\title{
INFLUÊNCIA DAS CONDIÇÕES DO SOLO NA CLIMATOLOGIA DA PREVISÃO SAZONAL DO MODELO ETA
}

\author{
RESENDE, Nicole - nicole.resende@yahoo.com.br \\ Instituto Nacional de Pesquisas Espaciais \\ CHOU, Sin Chan - chou.sinchan@cptec.inpe.br \\ Instituto Nacional de Pesquisas Espaciais
}

\begin{abstract}
RESUMO: Modelos numéricos têm importante papel na previsão de tempo e clima, amparando assim as tomadas de decisões em diversos setores. O aprimoramento destes modelos pode ser feito a partir de parametrizações de processos de superfície, como o balanço de umidade do solo. Esse balanço é obtido a partir de parâmetros hidrológicos definidos para cada tipo de solo, e pela umidade inicial inserida no modelo. O objetivo desse trabalho é estudar a influência das condições iniciais do solo e do tipo de solo na previsão sazonal do modelo Eta. São realizados dois experimentos (ETA1 e ETA2) a partir de integrações do modelo Eta, com 4 meses e meio de integração e utilizando reanálises como condições inicial e de contorno. 0 período analisado são as estações secas e chuvosas no Brasil, entre 1979 e 2012. ETA1 foi realizado como uma simulação controle, com umidade inicial do solo climatológica e nove tipos de solo. Em ETA2, utilizou-se umidade inicial do solo calculada e 26 tipos de solo. Para analisar a climatologia simulada, dados de reanálises permitiram a avaliação dos erros de algumas variáveis do modelo. A partir da análise dos experimentos, conclui-se que o modelo Eta manifesta sensibilidade a alterações no solo quando diferentes condições de solo são utilizadas na integração do modelo. ETA2 simula temperaturas sistematicamente menores e precipitação mais acurada em algumas regiões do Brasil.
\end{abstract}

Palavras-chave: Modelo Eta, condições do solo, reanálises, precipitação, temperatura.

INFLUENCE OF CONDITIONS OF SOIL IN SEASONAL ETA MODEL FORECAST

ABSTRACT: Numerical models have important role in predicting weather and climate, helping decision making in various sectors. The improvement of these models can be made from parameterization of surface processes, such as soil moisture balance. This balance is obtained from hydrological parameters defined for each soil type, and by initial moisture inserted in the model. The propose of this work is study the influence of initial conditions of soil and type of soil in seasonal Eta model forecast. Two experiments are performed from Eta Model integrations (ETA1 and ETA2), with 4 and a half months of integration and using reanalysis as initial and boundary conditions. The analysis period is the dry and rainy seasons in Brazil between 1979 and 2012. ETA1 was performed as a control simulation with climatological initial soil moisture and 9 types of soil. In ETA2, we used soil water content calculated and 26 soil types. For analyze the simulated climatology, reanalysis data it was used to evaluation of the errors of some variables in the model. From the analysis of the experiments, it is concluded that Eta model expresses sensitivity when different initial soil conditions are used in the model. ETA2 simulates lower temperature and rainfall more acurate in most regions of Brazil.

Keywords: Eta Model, soil conditions, reanalysis, precipitation, temperature.

\section{INTRODUÇÃO}

O Brasil é um país com grande extensão territorial, o que favorece a diversidade climática e evidencia a importância da obtenção de previsões meteorológicas de boa qualidade. Para tanto, o Centro de Previsão de Tempo e Estudos Climáticos do Instituto Nacional de Pesquisas Espaciais (CPTEC/INPE) utiliza modelos numéricos, que tem importante papel na previsão de tempo e clima, amparando assim as tomadas de decisões em diversos setores. No entanto, há ainda algumas limitações no uso de previsões climáticas aplicadas ao setor elétrico que ocorrem, em grande parte, devido às incertezas das atuais previsões climáticas, relacionadas as simplificações e parametrizações necessárias aos modelos de previsão. Ao realizar uma previsão 
numérica, possivelmente serão encontrados erros sistemáticos associados a essas imperfeições do modelo, como por exemplo, uma tendência do modelo em subestimar ou superestimar certa variável em determinada região. Essa problemática torna essencial a realização de trabalhos com o intuito de verificar as regiões onde esses erros sistemáticos são mais proeminentes.

Diversos estudos têm sido realizados com modelos regionais com o objetivo de melhor representar os processos de mesoescala, topografia e características da superfície terrestre da América do Sul. Quando se trata de modelos regionais, a confiabilidade das simulações em alta resolução depende da qualidade da condição de contorno lateral e também da própria capacidade do modelo regional de desenvolver características realísticas do clima presente (SILVA, 2012). Apesar das limitações da modelagem climática regional, tais como as condições de contorno laterais, inicialização, o tempo de spin-up do modelo, entre outras, os modelos regionais apresentam diversos benefícios em sua utilização (GIORGI e MEARNS, 1999; TANAJURA, 1996; DICKINSON et al., 1989). Uma das vantagens do uso de modelos regionais é que suas grades incorporam uma alta resolução de topografia (não implícita na modelagem de grande escala) que tendem a representar melhor os efeitos da orografia na distribuição de chuva intra-regional da área de interesse modelada. Porém, em uma escala sazonal, os modelos de previsão regional possuem deficiências em suas simulações, devido ao comportamento caótico da atmosfera e a dificuldade em simular as condições atmosféricas após um determinado período.

As simulações de tempo e clima também são diretamente influenciadas pelas condições do solo e pela umidade inicial inserida em um modelo de previsão, devido à influência destes na partição de energia entre calor latente e calor sensível (DIRMEYER et al, 1999). Essa partição é responsável pelas diferenças na disponibilidade de calor e água na superfície, determinando o balanço de energia na superfície e o ciclo hidrológico da região. Verifica-se então que as condições do solo podem afetar não só a temperatura próxima da superfície e a umidade do ar, mas também a circulação local e a precipitação. Isso mostra a importância da realização de estudos que investiguem a sensibilidade de modelos atmosféricos às variações de umidade do solo.

O objetivo principal deste trabalho é estudar a influência das condições iniciais do solo e do tipo de solo na previsão sazonal, auxiliando o desenvolvimento do modelo Eta através da avaliação do seu desempenho para a previsão sazonal. Busca-se também avaliar se o modelo é capaz de capturar variabilidade climática interanual e inferir como se comportam os erros sistemáticos das saídas do modelo durante as estações secas e chuvosas no Brasil.

\section{METODOLOGIA}

\subsection{Modelo de previsão Eta/CPTEC}

Neste trabalho é utilizado o modelo de previsão sazonal Eta/CPTEC, com resolução de $15 \mathrm{~km}$. Com relação à dinâmica do modelo, sua topografia é representada em forma de degraus discretos, cujo topo coincide com a interface das camadas (MESINGER et al, 2012). O modelo utiliza coordenada vertical Eta, representada pela letra grega $\eta \mathrm{e}$ descrita por Mesinger (1984) como uma pressão de referência adequadamente definida em função da altura, conforme equações (1 e 2) a seguir: 


$$
\eta=\left(\frac{P-P_{t}}{P_{s f c}-P_{t}}\right) \eta_{s f c}
$$

onde,

$$
\eta_{s f c}=\frac{P_{r e f}\left(Z_{s f c}\right)-P_{t}}{P_{r e f}(0)-P_{t}}
$$

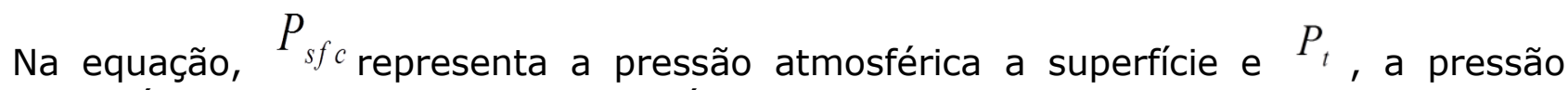
atmosférica no topo da atmosfera. O índice ref se refere a pressão em uma atmosfera de referência e $Z_{s f c}$ é a altitude da superfície.

A coordenada $\eta$ foi desenvolvida para reduzir erros no cálculo de derivadas horizontais, principalmente as forças de gradiente de pressão perto de montanhas, pois, considerando a superfície dessa coordenada aproximadamente horizontal, as variáveis obtidas através de derivadas horizontais são melhor representadas. Em regiões montanhosas, o topo das montanhas coincidem com as coordenadas e é justamente nessas regiões íngremes que a redução do erro é mais significativa. Maiores detalhes sobre a dinâmica do modelo Eta podem ser obtidos em Mesinger et al., 2012. Outras configurações do modelo são dispostas na Tabela 1.

O tempo de integração da versão do modelo Eta sazonal é de quatro meses e meio, no entanto, foram utilizados apenas os dados dos quatro meses completos. Para a realização desse trabalho foi escolhido um período representativo de clima úmido para o Brasil, compreendido de novembro a fevereiro (NDJF), e outro período representativo de clima seco, neste trabalho considerado como os meses de maio a agosto (MJJA). Na estação chuvosa, a integração do modelo teve início no dia 13 de outubro, e na estação seca, no dia 13 de abril. Essa análise foi realizada para os anos de 1979 a 2012, sendo que, este período foi escolhido devido a disponibilidade de dados de reanálises (nesse trabalho utilizados como condição inicial e de contorno para as simulações).

Tabela 1. Configurações e parametrizações utilizadas no modelo Eta.

\begin{tabular}{l|l}
\hline Domínio & Brasil \\
\hline Variáveis prognósticas & $\begin{array}{l}\text { Temperatura, vento (componentes zonal e } \\
\text { meridional), pressão à superfície, energia cinética } \\
\text { turbulenta e hidrometeoros das nuvens }\end{array}$ \\
\hline Grade do modelo (im x jm x Im) & $237 \times 435 \times 38$ \\
\hline $\begin{array}{l}\text { Grade pós - processada (lat x lon x } \\
\text { níveis de pressão) }\end{array}$ & $320 \times 273 \times 20$ \\
\hline Ponto central & $58^{\circ}$ W; $15^{\circ} \mathrm{S}$ \\
\hline Resolução horizontal & 15 km \\
\hline Grade horizontal & Grade E de Arakawa (ARAKAWA e LAMB, 1977) \\
\hline Prazo de previsão & 4,5 meses \\
\hline Processos turbulentos & $\begin{array}{l}\text { Atmosfera livre: esquema de Mellor-Yamada nível 2.5 } \\
\text { (MELLOR e YAMADA, 1982). Camada superficial: } \\
\text { funções de estabilidade de Paulson (PAULSON, 1970). }\end{array}$ \\
\hline Esquema de integração & Particionamento explícito ('split-explicit') \\
\hline Esquema de convecção & Betts-Miller-Janjic (JANJIC, 1994) \\
\hline
\end{tabular}




\begin{tabular}{l|l}
\hline Precipitação estratiforme & $\begin{array}{l}\text { Esquema de microfísica de nuvens de Zhao (ZHAO e } \\
\text { CARR, 1997). }\end{array}$ \\
\hline Radiação & $\begin{array}{l}\text { Esquema GFD (Geophysical Fluid Dynamics } \\
\text { Laboratory), com ondas longas e ondas curtas } \\
\text { parametrizadas de acordo com Fels e Schwarzkopf } \\
(1975) \text { e Lacis e Hansen (1974), respectivamente. }\end{array}$ \\
\hline Mapa de vegetação & PROVEG - INPE \\
\hline Esquema de superfície & NOAH (EK et al, 2003; CHEN et al.,1997) \\
\hline $\begin{array}{l}\text { Dióxido de carbono, ozônio e albedo } \\
\text { inicial }\end{array}$ & Retirados da climatologia \\
\hline
\end{tabular}

\subsection{Condições iniciais e de contorno utilizadas}

Dados de reanálises constituem um recurso importante e amplamente utilizado para o estudo da previsibilidade dos processos atmosféricos e oceânicos. Esses são cada vez mais utilizados em campos que necessitam de um registro de observação contínua do estado da atmosfera. Uma aplicação particular desses dados está relacionada à validação de simulações de longo prazo de modelos numéricos, desenvolvendo assim a capacidade de previsão sazonal.

Neste estudo, foram empregados como condição inicial e condição de borda lateral para o modelo Eta os dados da reanálise Climate Forecast System Reanalysis (CFSR), para o período de 1979 a 2012. Conhecido como CFSR e provenientes do Nacional Center for Environmental Prediction (NCEP) estes dados possuem resolução de $0.5^{\circ}$, com 64 níveis que se estendem a partir da superfície até 0,26 hPa. A condição de contorno lateral também é dada pelas reanálises do CFSR, com frequência de atualização a cada 6 horas. Mais informações a cerca desses dados podem ser obtidas em Saha et. al (2010).

Algumas variáveis necessárias para a iniciação do modelo não estão disponíveis nas reanálises CFSR, então para suprir a necessidade desses dados, utilizou-se uma climatologia da reanálise ERA Interim como uma informação complementar. A reanálise ERA Interim é a reanálise atmosférica global mais recente produzida pelo European Centre for Medium-Range Weather Forecasts (ECMWF). Maiores informações sobre essas reanálises podem ser obtidas em Dee et al (2011).

As variáveis necessárias como condição inicial e de contorno do modelo, bem como suas unidades e fontes, são descritas na Tabela 2.

O conteúdo de umidade do solo também é uma variável de entrada no modelo, contudo, esta foi descrita na seção seguinte, pois varia nos experimentos. 
Tabela 2. Variáveis utilizadas como condição inicial e de contorno para o modelo Eta.

\begin{tabular}{l|l|l|l}
\hline SIGLA & DESCRIÇÃO DA VARIÁVEL & UNIDADE & FONTE \\
\hline PsIm & Pressão ao nível médio do mar & $\mathrm{hPa}$ & CFSR \\
Prec & Total acumulado em 6 horas de precipitação & $\mathrm{M}$ & CFSR \\
Tgsc & Temperatura do solo a 0,1 metros & $\mathrm{K}$ & ERA Interim \\
Tgrz & Temperatura do solo a 2 metros & $\mathrm{K}$ & ERA Interim \\
Zgeo & Altura geopotencial & Metros & CFSR \\
Uvel & Vento zonal & $\mathrm{m} / \mathrm{s}$ & CFSR \\
Vvel & Vento meridional & $\mathrm{m} / \mathrm{s}$ & CFSR \\
Temp & Temperatura & $\mathrm{m} / \mathrm{s}$ & CFSR \\
Umrl & Umidade relativa & $\%$ & CFSR \\
Tsm & Temperatura da superfície do mar & $\mathrm{K}$ & ERA Interim \\
\hline
\end{tabular}

\subsection{Configurações dos experimentos}

A umidade do solo é uma variável determinante nos processos hidrológicos, influenciando nas interações atmosfera-solo e na relação precipitação/escoamento. Para analisar a influência da umidade do solo sobre as simulações do modelo Eta, foram realizados dois experimentos, que consideram diferentes condições iniciais do solo, descritos como ETA1 e ETA2.

Os tipos de solo oferecem informações básicas que são incluídas em cada perfil. Funções de pedotransferência são aplicadas para obter os diferentes parâmetros hidrológicos que definem cada tipo de solo (TOMASELLA e HODNETT., 2005). Em ambos os experimentos, o tipo de solo foi definido a partir de alguns parâmetros hidrológicos, descritos na Tabela 3.

Tabela 3. Parâmetros utilizados para definir o tipo de solo.

\begin{tabular}{l|l}
\hline Siglas & Parâmetros hidrológicos \\
\hline MAXSMC & Valor da saturação da umidade do solo \\
SATPSI & Potencial de saturação do solo \\
SATDK & Condutividade hidráulica do solo saturado \\
BB & Parâmetro B do tipo de solo \\
QTZ & Quantidade de quartzo no solo \\
REFSMC & Limiar a partir do qual a umidade do solo começa a controlar a \\
WLTMSC & Valor volumétrico do ponto de murcha permanente \\
DRYSMC & Limiar para solo seco quando acaba de evaporar \\
SATDW & Difusividade do solo saturado \\
F11 & Relação entre difusividade e condutividade
\end{tabular}

Fonte: Adaptado de Tomasella e Hodnett (2005)

\subsubsection{Experimento 1. Parâmetros de solo definidos por Zobler (1986)}

No experimento 1 (ETA1) não foram realizadas modificações nas atuais configurações da versão sazonal do modelo. A umidade inicial utilizada, provêm da climatologia da reanálise ERA - Interim, comumente empregada nas simulações do modelo Eta. Essa climatologia fornece informações do conteúdo de umidade em 4 camadas de solo ( 0 a $7 \mathrm{~cm}, 7$ a $28 \mathrm{~cm}, 28$ a $100 \mathrm{~cm}$ e 100 a $255 \mathrm{~cm}$ ), em metro cúbico por metro cúbico 
$\left(\mathrm{m}^{3} / \mathrm{m}^{3}\right)$. Nesse experimento, foi utilizado o mapa de solo de Zobler (1986), que apresenta nove tipos de solo e resolução horizontal de $1^{\circ} \times 1^{\circ}$. Esses dados representam um subconjunto do arquivo do Mundo Zobler de Modelagem Climática Global (ZOBLER, 1986) e foram compilados como parte de um esforço para melhorar a modelagem do ciclo hidrológico de parte dos modelos climáticos globais. Os valores dos parâmetros hidrológicos que definiram o tipo de solo, seguem na Tabela 4.

Tabela 4 - Valores dos parâmetros hidrológicos, segundo metodologia de Zobler (1986).

\begin{tabular}{c|c|c|c|c|c|c|c|c|c|c}
\hline TIPO & MAXSMC & SATPSI & SATDK & BB & QTZ & REFSMC & WLTMSC & DRYSMC & SATDW & F11 \\
\hline 1 & 0,42 & 0,04 & $1,41.10^{-5}$ & 4,26 & 0,82 & 0,28 & 0,03 & 0,03 & $5,71.10^{-6}$ & $-1,00$ \\
2 & 0,46 & 0,62 & $0,20.10^{-5}$ & 8,72 & 0,10 & 0,39 & 0,12 & 0,12 & $2,33.10^{-5}$ & $-1,12$ \\
3 & 0,47 & 0,47 & $0,10.10^{-5}$ & 11,55 & 0,25 & 0,41 & 0,14 & 0,14 & $1,16.10^{-5}$ & $-2,14$ \\
4 & 0,43 & 0,14 & $0,52 \cdot 10^{-5}$ & 4,74 & 0,60 & 0,31 & 0,05 & 0,14 & $7,95.10^{-6}$ & $-0,57$ \\
5 & 0,41 & 0,10 & $0,72 \cdot 10^{-5}$ & 10,73 & 0,52 & 0,34 & 0,10 & 0,10 & $1,90.10^{-5}$ & $-3,20$ \\
6 & 0,47 & 0,26 & $0,25.10^{-5}$ & 8,17 & 0,35 & 0,38 & 0,10 & 0,10 & $1,14.10^{-5}$ & $-1,30$ \\
7 & 0,40 & 0,14 & $0,45 \cdot 10^{-5}$ & 6,77 & 0,60 & 0,32 & 0,07 & 0,07 & $1,06.10^{-5}$ & $-1,52$ \\
8 & 0,44 & 0,36 & $0,34 \cdot 10^{-5}$ & 5,25 & 0,40 & 0,33 & 0,07 & 0,07 & $1,46.10^{-5}$ & $-0,33$ \\
9 & 0,42 & 0,04 & $1,41.10^{-5}$ & 4,26 & 0,82 & 0,28 & 0,03 & 0,03 & $5,71.10^{-6}$ & $-1,00$ \\
\hline
\end{tabular}

Fonte: Adaptado de Zobler (1986).

\subsubsection{Experimento 2. Parâmetros definidos por Tomasella e Hodnett (2005)}

Nesse experimento o mapa de solo foi atualizado com a finalidade de analisar de que forma as condições iniciais do solo podem afetar as previsões de temperatura e precipitação de uma dada região. O mapa de solo utilizado nesse trabalho foi desenvolvido por Tomasella e Hodnett (2005), elaborado a partir de informações do solo disponíveis na base de dados do INPE. Esse inclui informações sobre perfis de solo do Brasil, Uruguai, Paraguai e Argentina, com base nas informações sobre perfis do solo da base de dados do CPTEC/INPE. A combinação desses parâmetros resultou em um mapa com 26 tipos de solos, distribuídos em uma grade de $0,25^{\circ} \times 0,25^{\circ} \mathrm{e}$, para completar as regiões onde não foi possível obter informações, foi utilizado um mapa de solo desenvolvido pelo National Center for Atmospheric Research (NCAR). O mapa com 26 tipos de solo é apresentado na Figura 1.

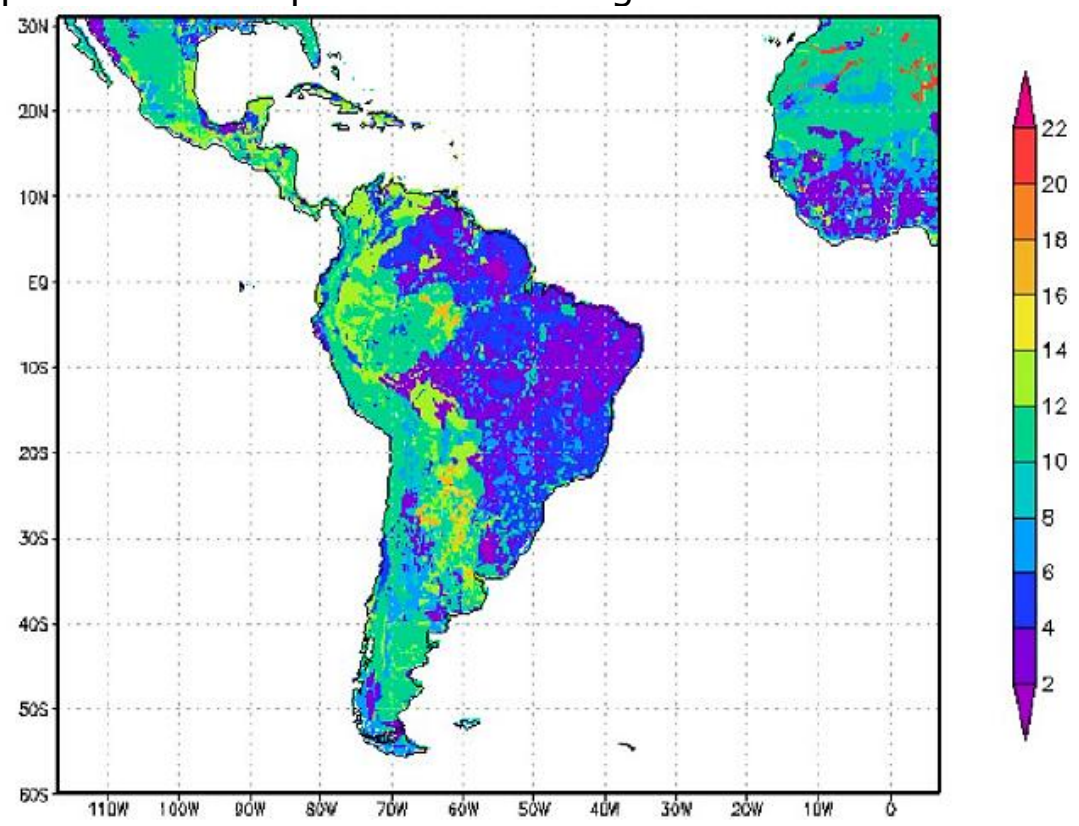

Figura 1. Mapa de solos incorporado no modelo Eta (com 26 tipos de solo) / Fonte: Tomasella e Hodnett (2005). 
Da mesma forma que o mapa de Zobler (1986), esse mapa foi desenvolvido a partir de 10 parâmetros sobre os quais foram definidos os tipos de solo. Os valores desses parâmetros seguem na Tabela 5 .

As condições iniciais de umidade do solo para a simulação, foram obtidas através de um modelo de balanço hídrico que atua de forma operacional no INPE. Este modelo utiliza precipitação diária obtida a partir do produto Tropical Rainfall Measuring Mission (TRMM), e precipitação observada coletada por pluviômetros de diferentes instituições e analisada pelo CPTEC (ROZANTE et al, 2010).

Os dados de umidade inicial do solo foram cedidos pelo CPTEC/INPE. São demonstrados a seguir como o modelo de balanço hídrico calcula a umidade inicial do solo em um esforço para uma representação mais realista da umidade do solo e seu impacto sobre a modelagem climática. É importante ressaltar que, ao analisar a umidade calculada para o período anterior a 1997, esta levava em conta dados de nebulosidade, tendo em vista a ausência de dados de radiação disponíveis para este período. No entanto, os dados de umidade a partir de 1997 consideram a radiação e esta diferença na metodologia pode apresentar uma certa discrepância nos resultados.

Tabela 5. Valores dos parâmetros hidrológicos, segundo Tomasella e Hodnett (2005).

\begin{tabular}{|c|c|c|c|c|c|c|c|c|c|c|}
\hline \multirow{2}{*}{ TIPO } & \multirow{2}{*}{\multicolumn{2}{|c|}{\begin{tabular}{|l|l} 
MAXSMC & SATPSI \\
\end{tabular}}} & \multirow{2}{*}{\multicolumn{2}{|c|}{\begin{tabular}{l|l} 
SATDK & BB \\
\end{tabular}}} & \multirow{2}{*}{ QTZ } & \multirow{2}{*}{\multicolumn{2}{|c|}{ WLTMSC }} & \\
\hline & & & & & & & & DRYSMC & SATDW & F11 \\
\hline 1 & 0,16 & 0,27 & $4,28 \cdot 10^{-7}$ & 2,03 & 0,60 & 0,03 & 0,08 & 0,08 & $1,48.10^{-6}$ & $-0,21$ \\
\hline 2 & 0,19 & 0,10 & $8,22 \cdot 10^{-7}$ & 2,78 & 0,25 & 0,04 & 0,16 & 0,16 & $1,21.10^{-6}$ & $-1,00$ \\
\hline 3 & 0,19 & 0,25 & $1,69.10^{-6}$ & 3,03 & 0,25 & 0,07 & 0,32 & 0,32 & $6,68 \cdot 10^{-6}$ & $-0,77$ \\
\hline 4 & 0,29 & 0,11 & $1,40 \cdot 10^{-5}$ & 2,25 & 0,60 & 0,05 & 0,11 & 0,11 & $1,16.10^{-5}$ & $-0,17$ \\
\hline 5 & 0,29 & 0,09 & $9,45 \cdot 10^{-5}$ & 2,62 & 0,45 & 0,06 & 0,19 & 0,19 & $7,60.10^{-6}$ & $-0,47$ \\
\hline 6 & 0,30 & 0,05 & $9,18 \cdot 10^{-5}$ & 3,02 & 0,25 & 0,06 & 0,27 & 0,27 & $4,90 \cdot 10^{-6}$ & $-0,88$ \\
\hline 7 & 0,37 & 0,09 & $7,70 \cdot 10^{-5}$ & 1,97 & 0,82 & 0,04 & 0,07 & 0,07 & $3,85 \cdot 10^{-5}$ & 0,13 \\
\hline 8 & 0,38 & 0,04 & $3,43 \cdot 10^{-5}$ & 2,53 & 0,25 & 0,05 & 0,18 & 0,18 & $9,35 \cdot 10^{-6}$ & $-0,45$ \\
\hline 9 & 0,36 & 0,02 & $5,02 \cdot 10^{-5}$ & 2,98 & 0,25 & 0,05 & 0,27 & 0,27 & $7,32 \cdot 10^{-6}$ & $-1,05$ \\
\hline 10 & 0,32 & 0,43 & $1,71.10^{-5}$ & 2,13 & 0,60 & 0,09 & 0,12 & 0,12 & $4,85 \cdot 10^{-5}$ & 0,58 \\
\hline 11 & 0,32 & 0,40 & $5,28 \cdot 10^{-6}$ & 2,44 & 0,35 & 0,10 & 0,20 & 0,20 & $1,61 \cdot 10^{-5}$ & 0,40 \\
\hline 12 & 0,30 & 0,22 & $3,46.10^{-6}$ & 2,90 & 0,25 & 0,10 & 0,26 & 0,26 & $7,22 \cdot 10^{-6}$ & $-0,17$ \\
\hline 13 & 0,37 & 0,60 & $2,05 \cdot 10^{-6}$ & 2,01 & 0,60 & 0,12 & 0,11 & 0,11 & $6,77 \cdot 10^{-5}$ & 0,91 \\
\hline 14 & 0,37 & 0,50 & $6,96 \cdot 10^{-6}$ & 2,42 & 0,10 & 0,13 & 0,20 & 0,20 & $2,30 \cdot 10^{-5}$ & 0,65 \\
\hline 15 & 0,40 & 0,13 & $4,95.10^{-6}$ & 2,97 & 0,10 & 0,11 & 0,28 & 0,28 & $4,95 \cdot 10^{-5}$ & $-0,05$ \\
\hline 16 & 0,31 & 0,93 & $3,64 \cdot 10^{-6}$ & 2,81 & 0,10 & 0,16 & 0,20 & 0,20 & $3,06 \cdot 10^{-5}$ & 0,54 \\
\hline 17 & 0,42 & 1,29 & $1,06.10^{-6}$ & 1,84 & 0,25 & 0,18 & 0,12 & 0,12 & $6,05 \cdot 10^{-5}$ & 1,41 \\
\hline 18 & 0,39 & 0,84 & $6,55 \cdot 10^{-6}$ & 2,30 & 0,10 & 0,16 & 0,19 & 0,19 & $3,28 \cdot 10^{-5}$ & 0,98 \\
\hline 19 & 0,48 & 0,76 & $2,80 \cdot 10^{-6}$ & 5,33 & 0,10 & 0,38 & 0,08 & 0,08 & $2,39 \cdot 10^{-5}$ & 0,16 \\
\hline 20 & 0,46 & 0,62 & $2,00 \cdot 10^{-6}$ & 8,72 & 0,10 & 0,39 & 0,12 & 0,12 & $2,37 \cdot 10^{-5}$ & $-1,12$ \\
\hline 21 & 0,47 & 0,32 & $1,30.10^{-6}$ & 10,4 & 0,10 & 0,40 & 0,13 & 0,13 & $9,60 \cdot 10^{-6}$ & $-1,92$ \\
\hline 22 & 0,20 & 0,07 & $1,41 \cdot 10^{-4}$ & 2,79 & 0,07 & 0,17 & 0,01 & 0,01 & $1,36 \cdot 10^{-4}$ & $-1,11$ \\
\hline 23 & 0,47 & 0,49 & $1,00 \cdot 10^{-6}$ & 11,6 & 0,60 & 0,45 & 0,03 & 0,03 & $1,12 \cdot 10^{-5}$ & $-0,47$ \\
\hline 24 & 0,20 & 0,07 & $1,41 \cdot 10^{-4}$ & 2,79 & 0,52 & 0,17 & 0,01 & 0,01 & $1,36 \cdot 10^{-4}$ & $-0,47$ \\
\hline 25 & 0,34 & 0,07 & $1,10.10^{-6}$ & 2,79 & 0,92 & 0,24 & 0,01 & 0,01 & $6,00 \cdot 10^{-7}$ & $-0,47$ \\
\hline 26 & 0,42 & 0,04 & $1,41 \cdot 10^{-5}$ & 4,26 & 0,25 & 0,28 & 0,03 & 0,03 & $5,10.10^{-6}$ & $-1,04$ \\
\hline
\end{tabular}

Fonte: Adaptado de Tomasella e Hodnett (2005). 
A variação temporal do armazenamento de água no solo acima do ponto de murcha (S) é estimada, em milímetros, a partir da equação (3) do balanço:

$$
\frac{\partial S}{\partial t}=P-R E T-D D
$$

Tal que:

$$
\begin{aligned}
& \mathrm{P}=\text { taxa de precipitação }(\mathrm{mm} / \mathrm{dia}) \\
& \text { RET = evapotranspiração }(\mathrm{mm} / \mathrm{dia}) \\
& \mathrm{DD}=\text { taxa de drenagem profunda }(\mathrm{mm} / \mathrm{dia})
\end{aligned}
$$

A evapotranspiração real (RET) é função da evapotranspiração potencial (PET), do armazenamento de água no solo acima do ponto de murcha permanente (S) e de um teor de umidade crítica (CMC), conforme as equações 4 e 5.

$$
\begin{aligned}
& R E T=\frac{S}{C M C} \cdot P E T \text { se } S<C M C \\
& R E T=P E T \text { se } S \geq C M C
\end{aligned}
$$

CMC é o parâmetro do solo que define o limite de armazenamento de água do solo no qual as raízes das plantas podem receber água do solo, sem danos. Curvas características do solo, tais como quantidade de água a um determinado potencial matricial, definem esse parâmetro. A taxa de drenagem profunda é calculada através da equação (6) na qual se assume a drenagem realizada através da gravidade.

$$
D D=K_{s}\left(\frac{S}{S_{\text {max }}}\right)^{\eta}
$$

Tal que:

ks $=$ condutividade hidráulica do solo saturado.

Smax = teor máximo de umidade do solo.

$\eta$ = parâmetro de Brooks-Corey

Através dessas variações, o teor de umidade do solo total é obtido. Conforme a equação (7) a seguir, quando o armazenamento resultante é maior do que o conteúdo máximo de umidade do solo $\left(S_{\max }\right)$, o modelo considera este valor um excesso $\left(E X C_{\text {sat }}\right)$. Assim, o modelo ajusta o teor de umidade do solo igual ao teor máximo de umidade do solo.

se $S>S_{\max }$, então $E X C_{\text {sat }}=S-S_{\max }$

senão; $S=S_{\max }$

Os campos de umidade do solo resultantes correspondem a valores acima do ponto de murcha. Maiores informações sobre este novo mapa de solo podem ser encontradas no trabalho de Doyle et al. (2013). 


\subsection{Erros sistemáticos do modelo}

Ao analisar as saídas de um modelo assumimos o pressuposto de que esses dados, representam bem as condições climáticas sobre a região de estudo. Esse nem sempre é o caso, pois em algumas regiões o modelo atua bem, representando de forma realística as diferentes variáveis quando comparados a dados observados, no entanto, em outras regiões o modelo pode encontrar certas dificuldades na previsão devido a diversos fatores tais como topografia, vegetação, condição inicial e lateral utilizada, entre outros.

Sabe-se que a análise da climatologia de uma região auxilia na avaliação do clima em escala local. Sendo assim, para identificar os erros sistemáticos do modelo e avaliar a sua capacidade em capturar a variabilidade interanual, foi construída uma climatologia a partir dos dados simulados pelo modelo Eta, considerando os dois experimentos realizados, e também a partir dos dados de reanálises. Portanto, os erros sistemáticos foram verificados por meio da subtração da climatologia dos dados observados aos dados simulados pelos experimentos. Esta é uma análise importante pois, através do estudo dos erros sistemáticos do modelo, é possível inferir se o modelo tende a superestimar ou subestimar a previsão dessas variáveis na região de estudo. Sendo assim, é possível verificar se a resolução de $15 \mathrm{~km}$ do modelo apresentou uma boa previsão para o domínio do Brasil no período estudado, e em qual experimento a previsão apresentou melhores resultados.

Construiu-se a climatologia dos dados mensais de temperatura (média a 2 metros) e precipitação do Climatic Research Unit (CRU), Version 3.21, a qual possui dados a partir de 1901 até o presente (JONES e HARRIS, 2013). É importante ressaltar que, em algumas regiões, a confiabilidade destes dados é questionável pois, há poucas observações utilizadas para realizar as interpolações necessárias para criar os dados do CRU. Em todos os casos (experimentos e reanálises), a climatologia foi feita para os meses de novembro a fevereiro (NDJF) e maio a agosto (MJJA), para os anos de 1979 a 2012.

\section{RESULTADOS}

O mapa de solo, após interpolado para a grade do modelo, apresenta características diferentes na inicialização dos experimentos, conforme Figura 2.

Observa-se que no mapa de solo do Experimento 1 (ETA1), o domínio escolhido restringe o número de tipos de solo para apenas 5 tipos. No mapa de solo do Experimento 2 (ETA2), são encontrados 20 tipos de solos, ou seja, novamente há redução do número de tipos de solo em relação ao mapa original (26 tipos). Pode-se verificar ainda na Figura 2, o melhor detalhamento no mapa de solo do ETA2 em relação ao ETA1. Contudo, é importante ressaltar que a acurácia do modelo varia nos dois experimentos, devido ao mapa de solo diferenciado, e devido também a umidade inicial do solo utilizada em cada experimento. Na Figura 2, os números e cores corresponde a parametrização do solo, conforme Tabela 4 e 5. 
a) ZOBLER (1986)

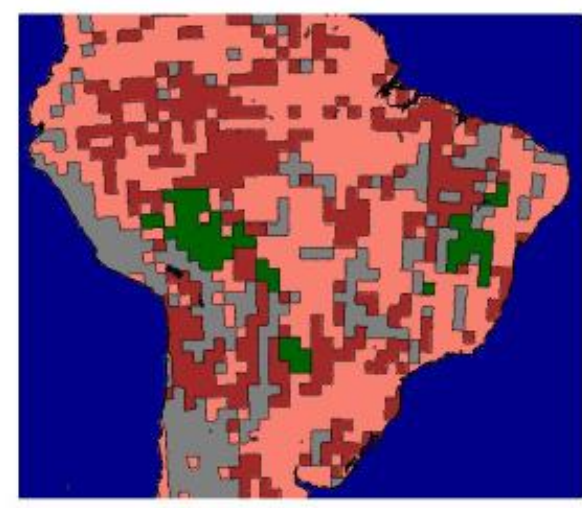

-

\section{b) TOMASELLA e HODNETT (2005)}

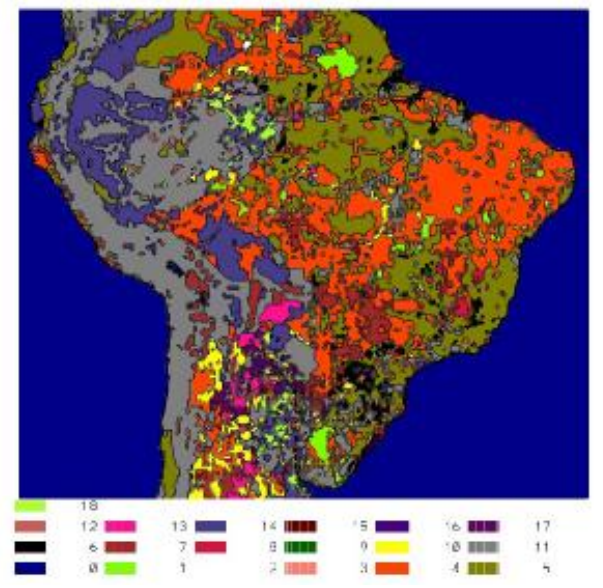

Figura 2. Tipos de solo interpolados para a grade do modelo Eta; a) ETA1 - Mapa com 9 tipos de solo (ZOBLER, 1986), b) ETA2 - Mapa com 26 tipos (TOMASELLA e HODNETT, 2005).

\subsection{Temperatura}

Com a mudança nas condições iniciais do solo, espera-se que haja uma variação da temperatura entre os experimentos, principalmente próximo à superfície. A Figura 3 exibe a climatologia da temperatura a 2 metros da superfície, simulada pelos experimentos nas estações secas e chuvosas.

$\mathrm{Na}$ Figura $3(\mathrm{a}, \mathrm{b})$, identifica-se que $\mathrm{o}$ experimento ETA2 tende a simular temperaturas reduzidas em relação ao ETA1 sobre todo o continente, na estação de MJJA. A temperatura simulada varia ao longo dos meses apenas na região próxima a linha do Equador, onde há aumento da temperatura com o decorrer dos meses da estação (não mostrado). Em relação a temperatura simulada na estação chuvosa, verifica-se nos items $c$ e $d$ da Figura 3 o mesmo padrão identificado na estação seca, ou seja, ETA2 simula temperaturas inferiores as simuladas por ETA1, em toda a região continental.

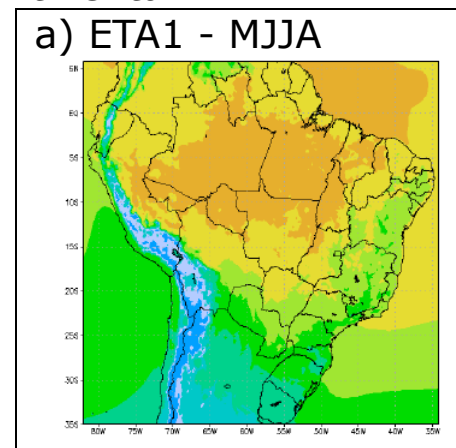

c) ETA1 - NDJF

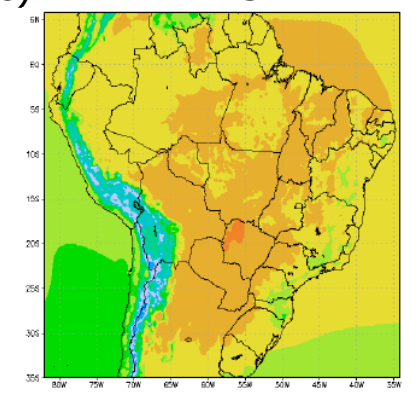

b) ETA2 - MJJA

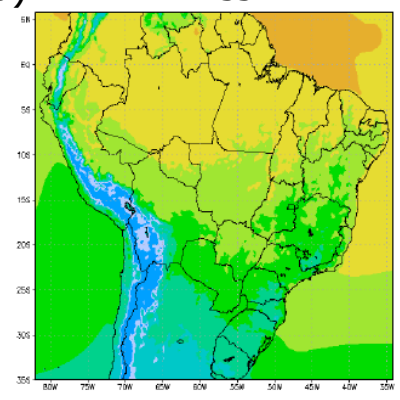

d) ETA2 - NDJF

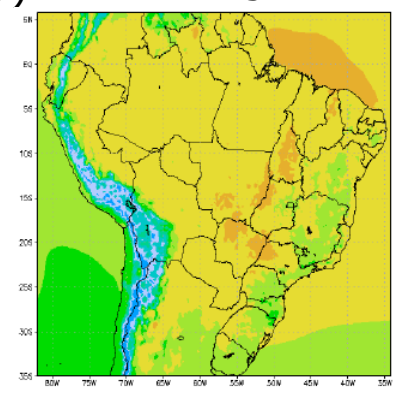

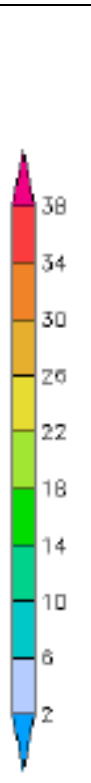

Figura 3. Temperatura a 2 metros da superfície simulada pelo modelo Eta $\left({ }^{\circ} \mathrm{C}\right)$, nos experimentos ETA1 $(a, c)$ e $\operatorname{ETA2}(b, d)$, nas estações secas (a, b) e chuvosas (c, d) de 1979 a 2012 
Os erros associados aos experimentos são expostos na Figura 4:

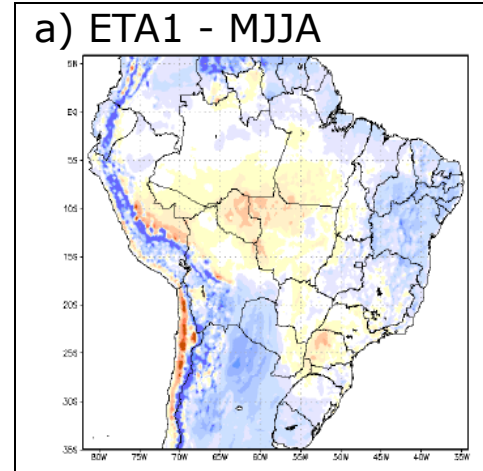

c) ETA1 - NDJF

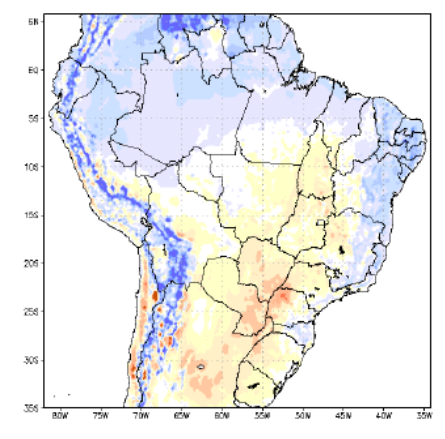

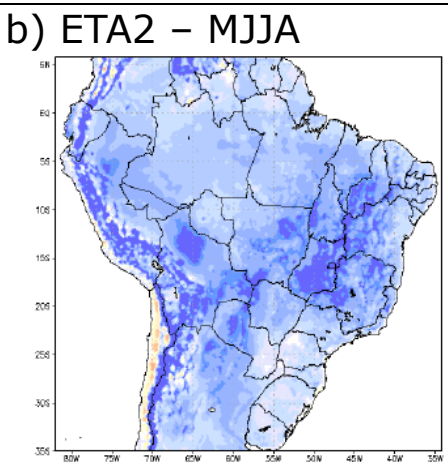

d) ETA2 - NDJF

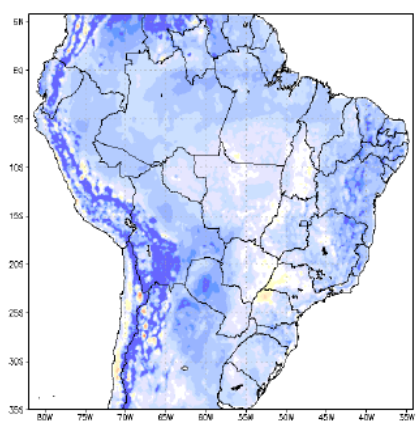

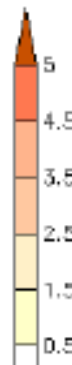

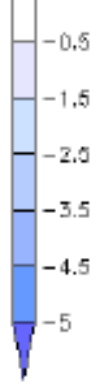

Figura 4. Erro da climatologia da temperatura a 2 metros da superfície $\left({ }^{\circ} \mathrm{C}\right)$, em relação ao $\mathrm{CRU}$, nos experimentos $\operatorname{ETA1}(a, c)$ e $\operatorname{ETA2}(b, d)$, nas estações secas (a, b) e chuvosas (c, d) de 1979 a 2012.

O experimento ETA1 apresenta erros variáveis na simulação da temperatura sobre o Brasil, com tendência a superestimar no norte e sul do Brasil, e subestimar principalmente sobre o nordeste brasileiro. Os erros na análise da climatologia mostram-se maiores no ETA2, indicando um padrão de subestimativa da temperatura em todo o domínio. Portanto, identifica-se a tendência do experimento ETA2 reduzir a temperatura (esfriar) em todo o continente. Esse padrão de subestimativa foi observado anteriormente por Vieira Jr. et al (2009), em relação as temperaturas máximas simuladas na região centro-sul do Brasil.

É importante ressaltar que não são encontradas variações no erro da simulação no decorrer dos meses de cada estação. No entanto, identifica-se que os maiores erros na simulação da temperatura a 2 metros da superfície estão associados a estação seca.

Estes resultados, em que a temperatura de ETA2 se mostra sistematicamente menor que a temperatura simulada por ETA1 estão diretamente relacionados à simulação da altura geopotencial em diferentes níveis. Ao analisar a altura geopotencial simulada nos dois experimentos, verifica-se que ambos os experimentos subestimam essa variável, indicando portanto, camadas frias. Em concordância com os dados expostos, verificou-se que a subestimativa da altura geopotencial é ainda maior no ETA2 (não mostrado).

\subsection{Precipitação}

Analisa-se a precipitação simulada nos dois experimentos (Figura 5), em milímetros acumulados nos 4 meses da estação seca (MJJA) e chuvosa (NDJF). 


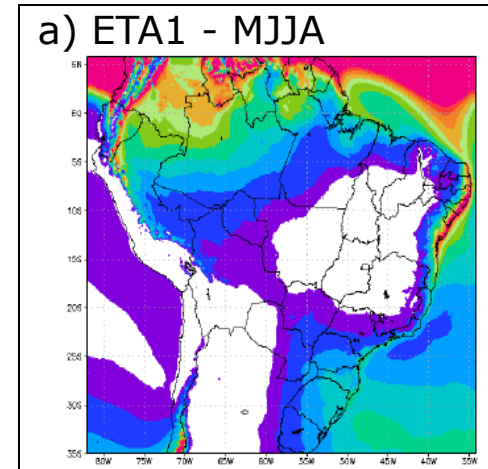

c) ETA1 - NDJF

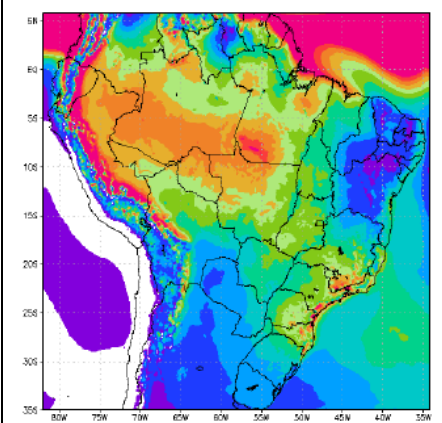

b) ETA2 - MJJA

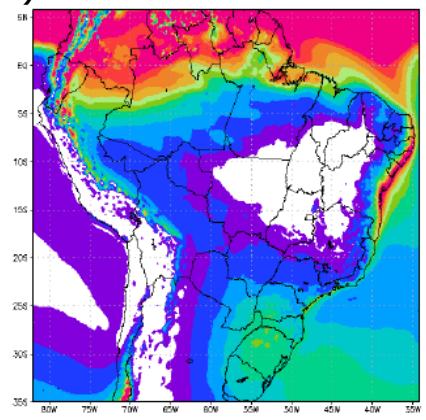

d) ETA2 - NDJF

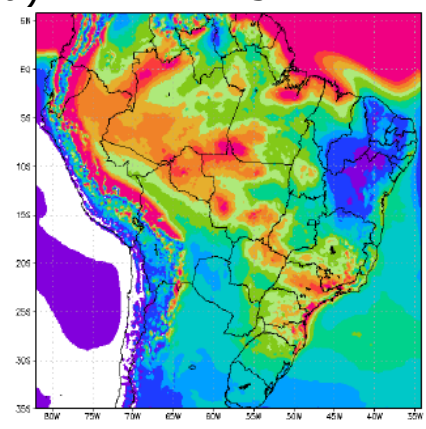

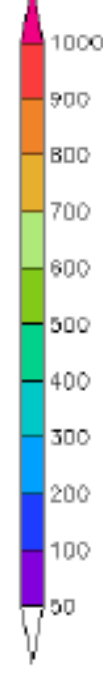

Figura 5. Precipitação simulada pelo modelo Eta (mm/estação), nos experimentos ETA1 (a, c) e ETA2 (b, d), nas estações secas (a, b) e chuvosas (c, d) de 1979 a 2012.

A partir da Figura 5 pode-se inferir que nas estações secas e chuvosas, o modelo é capaz de representar bem o padrão da estação.

Na estação seca, o interior do Brasil apresenta-se seco em ambos os experimentos, embora no ETA2 a simulação seja um pouco mais úmida. Os dois experimentos conseguem capturar a variabilidade interanual da precipitação, reduzindo o volume precipitado ao longo dos diferentes meses de MJJA (não mostrado). Contudo, ETA2 tende a gerar mais precipitação sobre o noroeste da América do Sul e sul do Brasil.

No período chuvoso (NDJF), ETA2 mantêm a tendência de gerar maior volume de precipitação que ETA1, principalmente nas regiões sul e central do Brasil. Pode-se concluir também que ETA2 representa melhor o padrão das ZCAS (Zona de Convergência do Atlântico Sul), fenômeno comum nessa época do ano, principalmente no mês de janeiro.

Na Figura 6, são dispostos os erros sistemáticos do modelo Eta, em relação aos dados do CRU: 


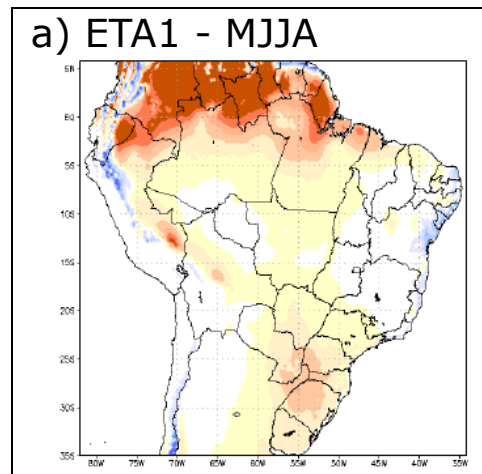

b) ETA1 - NDJF
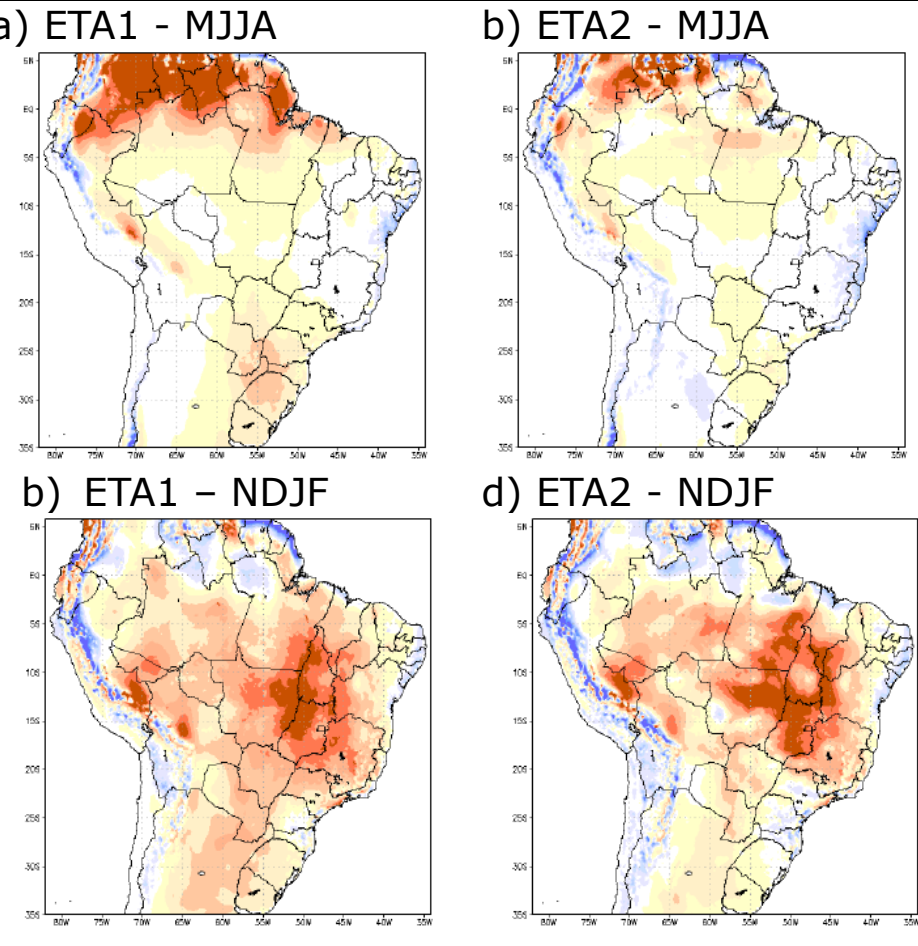

d) ETA2 - NDJF
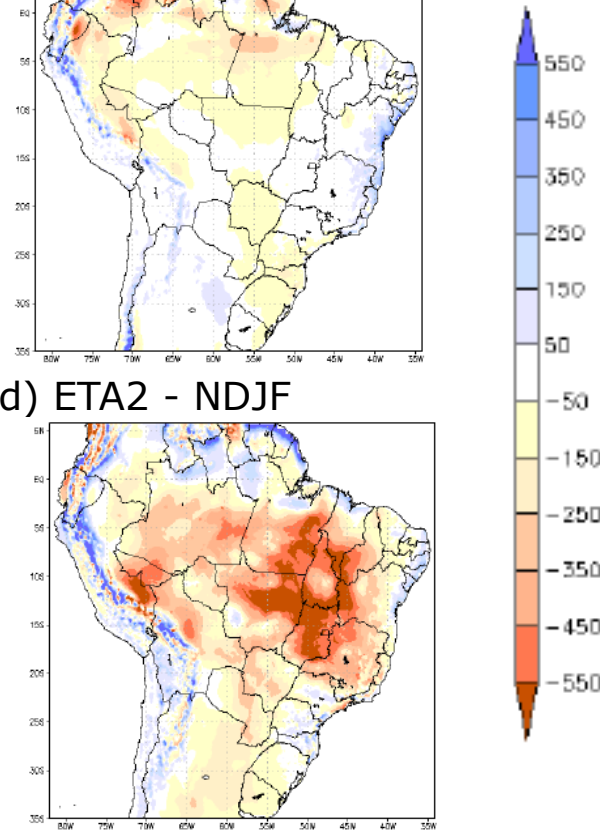

Figura 6. Erro da climatologia da precipitação (mm/estação), em relação ao CRU, nos experimentos ETA1 $(a, c)$ e $\operatorname{ETA2}(b, d)$, nas estações secas $(a, b)$ e chuvosas (c, d) de 1979 a 2012.

Observa-se que o ETA2 apresenta maior acurácia na simulação da precipitação no período de MJJA. No entanto, ETA2 agrava os erros encontrados no ETA1 na região litorânea do nordeste, região em que há grande tendência do modelo em superestimar a precipitação.

Ainda segundo a Figura 6, o ETA2 também apresenta melhoria na simulação da estação chuvosa em algumas regiões. Em relação aos dados do CRU, ETA2 mostra grande ganho na região sul do Brasil e norte da Argentina, locais em que observa-se subestimativa do ETA1. Sobre algumas regiões do norte, nordeste e centro-oeste do Brasil, há aumento na subestimativa da precipitação, fazendo com que ETA2 tenha menor acurácia que ETA1 nessas regiões. As simulações representam bem o regime de precipitação da América do Sul para a época analisada, com os meses de janeiro e fevereiro tendendo a ser mais chuvosos, em ambos os experimentos. ETA1 possui erros maiores em dezembro, e ETA2 em janeiro.

Nas diferentes estações, observa-se que a mudança nas condições do solo melhora a simulação de precipitação em parte do domínio estudado. Contudo, o experimento ETA2, na maior parte do domínio (em ambos experimentos e estações do ano) persiste a tendência do modelo em subestimar a precipitação. A estação com maiores erros é a estação chuvosa.

É importante destacar neste estudo, que em algumas regiões, o erro na simulação da precipitação ultrapassou $500 \mathrm{~mm}$. Isso indica a necessidade de estudos que possam contribuir com a modelagem climática, visando o desenvolvimento dos modelos climáticos para melhorar as simulações para estas regiões.

Os resultados para a estação chuvosa corroboram com o trabalho de Alves et al. (2000), em que conclui-se que a chuva de verão é subestimada pelo modelo Eta, principalmente na região de atuação das ZCAS sobre o Brasil central e norte do Nordeste. Em ambas as estações do ano os resultados dessa seção concordam com os 
resultados de Bustamante et al. (2006), trabalho sobre o qual verifica-se superestimativas do modelo Eta ao longo de algumas áreas costeiras ao sul do Chile e Nordeste do Brasil. Os resultados dispostos, também estão em acordo com os encontrados por Doyle et al. (2013), que infere que modificar as condições iniciais de umidade do solo e incorporar um novo mapa de solos com parâmetros hidráulicos mais representativos dos solos sulamericanos torna a previsão de chuvas no norte da Argentina mais acurada. A partir desses resultados confirma-se a hipótese de Chang e Wetzel (1991), os quais inferiram que: os limites resultantes do aquecimento diferencial devido a gradientes de umidade do solo pode ser um importante fator no desenvolvimento da convecção. Os resultados deste trabalho também se mostram coerentes com os resultados apresentados por Gevaerd e Freitas (2006), os quais mostraram que a implementação de campos de umidade do solo espacialmente heterogêneos em modelos atmosféricos causam impactos na qualidade da simulação.

\section{CONCLUSÕES}

Ao realizar experimentos, utilizando diferentes condições de solo na integração do modelo Eta, o modelo manifesta sensibilidade a alterações no solo, indicando potencial melhora na previsão sazonal. Essas condições são dadas a partir da alteração da umidade inicial do solo e dos parâmetros hidrológicos que definem o tipo de solo da região.

O modelo Eta, após alterada as condições iniciais do solo (ETA2), simula nas estações secas e chuvosas temperaturas sistematicamente menores. Em ambas as estações, a temperatura simulada em ETA1 e ETA2 apresentam valores muito distintos. ETA2 tende a subestimar muito a temperatura a dois metros da superfície em toda a região continental. A simulação da precipitação é mais acurada no ETA2, com diminuição da subestimativas nos períodos secos e chuvosos.

\section{REFERÊNCIAS}

ALVES, L. M.; MELO, A. B. C.; CHOU, S. C.; NOBRE, P. Estudo comparativo entre a precipitacao sazonal simulada pelo modelo ETA e observada sobre o Brasil. In: CONGRESSO BRASILEIRO DE METEOROLOGIA, 11., 2000, Rio de Janeiro (RJ). Anais... 2000. p. 10291035. CDROM. (INPE10156PRE/5684).

ARAKAWA, A.;. LAMB V. R. Computational design of the basic dynamical process of the UCLA general circulation model, Methods Comput. Rev. Geophys. Space Phys., v. 17, p. 173 265, 1977.

BUSTAMANTE, J. F.; GOMES, J. L.; CHOU, S. S.. 5-Years Eta model seasonal forecast climatology over South America. Proceedings.. 8 ICSHMO, Foz do Iguaçu, Brazil, April 24-28, 2006, INPE, p. 503-506.

CHANG, JT.; WETZEL P. J.. Effects of spatial variations of soil moisture and vegetation on the evolution of a prestorm environment: A numerical case study. Mon Wea Rev. v. 119, p. 1368-1390, 1991.

CHEN, F.; JANJIC, Z.I.; MITCHELL, K. Impact of atmospheric surfacelayer parameterization in the new landsurface scheme of the NCEP mesoscale Eta model. Bound. Layer Meteor. v. 85, p. 391421, 1997.

DEE, D. P.; UPPALAA, S. M.; SIMMONSA, A. J.; BERRISFORDA, P.; POLIA, S. KOBAYASHIB, U.; ANDRAEC, M. A..; BALMASEDAA, G.; BALSAMOA, P.; BAUERA, P.; BECHTOLDA, A. C. M.; 
BELJAARSA, L;. VAN DE BERGD, J; BIDLOTA, N.; BORMANNA, C.; DELSOLA, R.; DRAGANIA, M.; FUENTESA, A. J.; GEERA, L.; HAIMBERGERE, S. B.; HEALYA, H.; HERSBACHA, E. V; HOLMA, L.; ISAKSENA, P. K.; LLBERGC, M.; KHLER, M.; MATRICARDI, A. P.; MCNALLY, B. M.; MONGESANZ, J. J.; MORCRETTE, B. K.; PARKG, C.; PEUBEYA, P.; ROSNAYA, C.; TAVOLATOE, J. N.. The ERAInterim reanalysis: configuration and performance of the data assimilation system . Q. J. R. Meteorol. Soc. v. 137, p. 553-597, 2011

DICKINSON, R. E.; R. M. ERRICO et al. A regional climate model for the western U.S. Climate Change, v. 15, p. 383422. 1989.

DIRMEYER, P. A.; DOLMAN, A. J.; SATO, N.. The pilot phase of the global soil wetness project. Bull Amer Meteor Soc. v. 80, p. 851-878, 1999.

DOYLE, M. E.; TOMASELLA, J.; RODRIGUEZ, D. A.; CHOU, S. C. Xperiments using new initial soil moisture conditions and soil map in the Eta model over La Plata Basin. Meteorology and Atmospheric Physics, v. 121, p. 119-136, 2013. doi: <10.1007/s0070301302655>.

EK, M. B.; MITCHELL, K. E.; LIN, Y.; ROGERS, E.; GRUMMEN, P.; KOREN, V.; GAYNO, G. e TARPLEY, J. D. Implementation of NOAH land surface advances in the National Centers for Environmental Prediction operational mesoscale Eta model. J. Geophys. Res., 108:8851, doi: 10.1029/2002JD003246, 2003.

FELS, S. B.; SCHWARZKOPF, M. D. The Simplified Exchange Approximation: A New Method for Radiative Transfer Calculations. J. Atmos. Sci., v. 32, p. 14751488, 1975.

GEVAERD, R; FREITAS, S. R. Estimativa operacional da umidade do solo para iniciação de modelos de previsão numérica da atmosfera. Parte I: Descrição da metodologia e validação. Revista Brasileira de Meteorologia, v. 21, n. 3a, p. 59-73, 2006.

GIORGI, F.; MEARNS, L. Introduction to special section: regional climate modeling revisited. J. Geophys. Res. v. 104, p.63356352, 1999.

JANJIC, Z. I. The step montain coordinate model: further developments of the convection, viscous sublayer, and turbulence closure schemes. Mon. Wheather Rev. 1994.

JONES, P.; HARRIS, I. CRU TS3.21: Climatic Research Unit (CRU) TimeSeries (TS) version 3.21 of high resolution gridded data of monthbymonth variatio in climate. Didcot, United Kingdom: NCAS British Atmospheric Data Centre, 2013.

LACIS, A. A.; HANSEN, J. E. A Parameterization of the Absorption of Solar Radiation in Earth"s Atmosphere. J. Atmos. Sci., v. 31, p. 118-133, 1974.

MELLOR, G. L.; YAMADA, T. Development of a turbulence closure model for geophysical fluid problems. Rev. Geophys. Space Phys., v. 20, p. 851875, 1982.

MESINGER, F. A blocking technique for representation of mountains in atmospheric models. Riv. Meteor. Aeronautica, v. 44, p. 195202, 1984.

MESINGER, F.; CHOU, S. C.; GOMES, J. L.; JOVIC, D.; BASTOS, P.; BUSTAMANTE, J. F.; LAZIC, L.; LYRA, A. A.; MORELLI, S.; RISTIC, I.; VELJOVIC, K.. An upgraded version of the Eta model. Meteorol Atmos Phys., v. 116, p. 63-79, DOI 10.1007/s007030120182z, 2012.

PAULSON, C. A. The mathematical representation of wind speed and temperature profiles in the unstable atmospheric surface layer. J. Appl. Meteorol., v. 9, p. 857861, 1970.

ROZANTE J. R.; MOREIRA D. S. Combining TRMM and Surface Observations of Precipitation: Technique and Validation over South America. Weather and Forecasting., v. 25, p. 885894, 2010. 
SAHA, S.; MOORTHI, S.; PAN, HL.; WU, X.; WANG, J.; NADIGA, S.; TRIPP, P.; KISTLER, R.; WOOLLEN, J.; BEHRINGER, D.; LIU, H.; STOKES, D.; GRUMBINE, R.; GAYNO, G.; WANG, J.; HOU, YT.; CHUANG, HY.; JUANG, HM. H.; SELA, J.; IREDELL, M.; TREADON, R.; KLEIST, D.; DELST, P. V.; KEYSER, D. ;DERBER, J.; EK, M.; MENG, J.; WEI, H.; YANG, R.; LORD, S.; DOOL, H. V. D.; KUMAR, A.; WANG, W.; LONG, C.; CHELLIAH, M.; XUE, Y.; HUANG, B.; SCHEMM, JK; EBISUZAKI, W.; LIN, R.; XIE, P.; CHEN, M.; ZHOU, S.; HIGGINS, W.; ZOU, CZ.; LIU, Q.; CHEN, Y.; HAN, Y.; CUCURULL, L.; REYNOLDS, R. W.; RUTLEDGE, G.; GOLDBERG, M.. The NCEP Climate Forecast System Reanalysis. American

Meteorological Society, 2010.

SILVA, W. L. Tendências Observadas e Projeções Futuras de Extremos Climáticos na Cidade do Rio de Janeiro. Monografia. Universidade Federal do Rio de Janeiro, Departamento de Meteorologia. XVIII, 85 p. Rio de Janeiro, 2012.

TANAJURA C. A. S. Modeling and analysis of the South American summer climate. Tese Doutorado University of Maryland, College Park Maryland. 1996.

TOMASELLA, J.; HODNETT, M. G. Pedrotransfer functions for tropical soils. In: Pachepsky Y.; Rawls, W. J. (Org.) Developments in soil science: pedotransfer functions in hydrology. 30 ed. Amsterdam: Elsevier, 2005, v. 30, p.415429.

VIEIRA Jr., P. A.; NETO, D. D.; CHOU, S. C.; MARTIN, T. N. Previsões meteorológicas do Modelo Eta para subsidiar o uso de modelos de previsão agrícola no CentroSul do Brasil Ciência Rural, Santa Maria, v. 39, p.412420, 2009.

ZHAO, Q.; CARR, F. H. A prognostic cloud scheme for operational NWP Models. Monthly Weather Review, v. 125, p. 19311953, 1997.

ZOBLER, L. A world soil file for global climate modelling. New York, New York, U.S.A. NASA Goddard Institute for Space Studies.1986. NASA Technical Memorandum. 1986. 